\title{
Triquetral fracture with associated pisiform subluxation
}

Lee Ping $\underline{G a n}^{1}$, MBBS, MRCS, Mala Satkunanantham ${ }^{1}$, MBBS, MRCS, Sechachalam Sreedharan ${ }^{1}$, MBBS, MRCS, Winston Yoon Chong $\underline{\mathrm{Chew}^{1}}$, MBBS, FRCSEd

\begin{abstract}
We herein present a case of right triquetral fracture with associated pisiform and flexor carpi ulnaris subluxation in a 29-year-old man. Initial radiography showed a right triquetral fracture. Computed tomography and magnetic resonance imaging demonstrated a triquetral fracture with a subluxated pisiform. Open reduction and lag screw fixation of the right triquetrum was performed, with good subsequent recovery of function. Although triquetral fracture with subluxation of the pisotriquetral joint is uncommon, attending clinicians should bear in mind the possibility of such an injury when managing traumatic ulnar-sided wrist pain.
\end{abstract}

Keywords: flexor carpi ulnaris subluxation, pisiform, pisotriquetral joint, triquetral fracture, ulnar-sided wrist pain

\section{INTRODUCTION}

Triquetral fractures are the second most common carpal bone fractures after scaphoid fractures.(1) Triquetral fractures can involve the dorsal rim, body, or avulsion of the volar aspect. ${ }^{(2)}$ A common mechanism of triquetral injury is hyperextension, causing an impaction shear-type fracture. ${ }^{(3)}$ Given the variety of attached ligaments to the triquetrum, significant associated soft tissue injuries can occur with a triquetral fracture. Herein, we describe a case of triquetral fracture presenting with subluxation of the pisotriquetral joint and flexor carpi ulnaris (FCU) tendon.

\section{CASE REPORT}

A 29-year-old male construction worker fell from height and landed on his right hand. He subsequently complained of pain over the right hypothenar region, which was exacerbated by gripping, with associated snapping of a tendon. He presented to the clinic two months post injury. Examination of the patient's right hand revealed the pisiform to be tender on palpation, and pisotriquetral grinding test was positive for pain. Together with the pisiform, the FCU tendon was subluxated upon ulnar deviation and dorsiflexion of the wrist, producing a visible snap. The triangular fibrocartilage complex (TFCC) was tender on palpation, but the distal radioulnar joint was stable. The subluxation was reduced on flexion of the wrist.

Radiography of the wrist in neutral position showed a fracture at the junction of the middle and lateral thirds of the triquetral body (Fig. 1), but no subluxation of the pisotriquetral joint was seen. Computed tomography (CT) confirmed the presence of a sagittal fracture involving the pisotriquetral joint (Fig. 2). Magnetic resonance $(M R)$ imaging further demonstrated the fracture site to be a thin rim of bone at the ulnar aspect of the pisotriquetral joint, resulting in instability of the joint and a tendency of the pisiform to sublux clinically (Fig. 3). As this fracture was unstable, surgery was performed to stabilise the fracture, which in turn stabilised the pisotriquetral joint and FCU tendon.

Under regional anaesthesia, wrist arthroscopy was first performed through the $3 / 4$ and $6 \mathrm{R}$ radiocarpal port sites, revealing

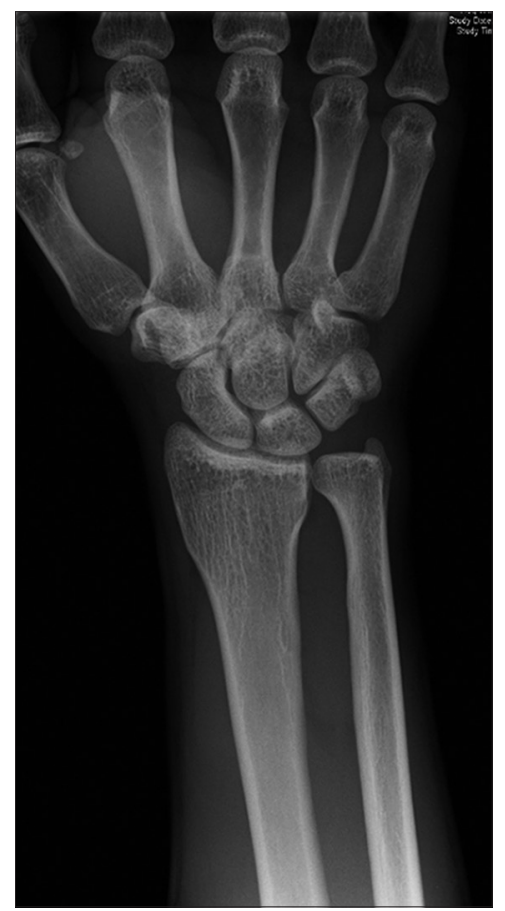

Fig. 1 Anteroposterior radiograph of the right wrist shows a fracture of the triquetral body.

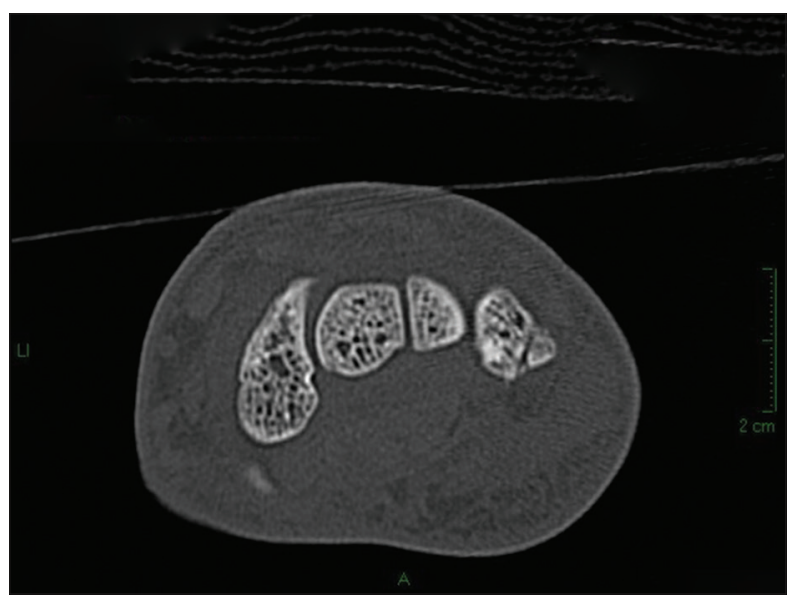

Fig. 2 CT image shows a fracture involving the pisotriquetral joint. 

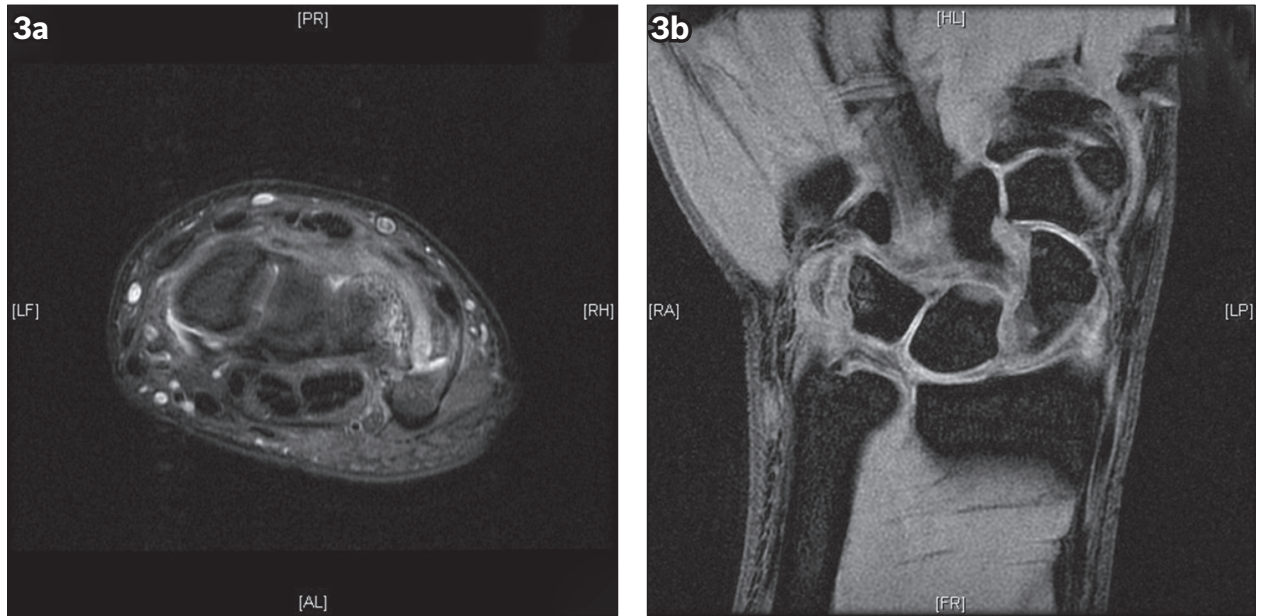

Fig. $3(a-b)$ MR images of the right wrist show a fracture at the ulnar aspect of the pisotriquetral joint.

a proximal partial tear of the scapholunate ligament, as well as a Palmer grade 1B TFCC tear. The TFCC tear was debrided and repaired via PDS 2/0 stitches. A zigzag incision was subsequently made over the volar-ulnar aspect of the wrist, and the FCU tendon was identified together with the pisiform. The pisotriquetral joint was partially opened proximally, and the fracture fragment was mobilised from the proximal and ulnar aspects. Intraoperative findings confirmed the diagnosis of right triquetral fracture, with instability and subluxation of the pisiform and fracture fragment over the triquetrum (Fig. 4). The fracture was initially reduced by being held with a K-wire and fixed with a 1.5-mm interfragmentary screw.

Postoperatively, the patient's right wrist was placed on a resting volar splint. He was started on intermittent range of motion (ROM) exercises one month post operation. There was no subluxation at the three-month follow-up, with only minimal pain on movement and exertion. The patient's ROM was as follows: right wrist palmar flexion at $40^{\circ}(66 \%$ of contralateral side); dorsiflexion at $60^{\circ}$ (85\% of contralateral side); ulnar deviation at $10^{\circ}(66 \%$ of contralateral side); radial deviation at $10^{\circ}(66 \%$ of contralateral side); and a grip strength of $22 \mathrm{~kg}$ ( $44 \%$ of contralateral side). The right triquetrum was stable, with no subluxation of the FCU tendon. The patient's pain was also noted to have improved. Postoperative radiography showed that the fracture had healed (Fig. 5). However, the patient was lost to subsequent follow-up, as he had returned to his native country. We expect his right wrist ROM to improve further.

\section{DISCUSSION}

The pisotriquetral joint describes the dorsal articulation between the pisiform and triquetrum. Flat and ovoid in shape, the joint derives its stability from soft tissue attachments such as the FCU tendon, ulnar pisotriquetral ligament, and the pisometacarpal and pisohamate ligaments. ${ }^{(4,5)}$ The pisotriquetral joint is tightly contained within both the transverse carpal ligament and the ulnar collateral ligament. ${ }^{(6)}$ Disruption of the pisotriquetral joint can occur secondary to direct trauma, or from traction caused by the FCU tendon either during a fall on a dorsiflexed wrist or while lifting heavy objects. ${ }^{(7,8)}$

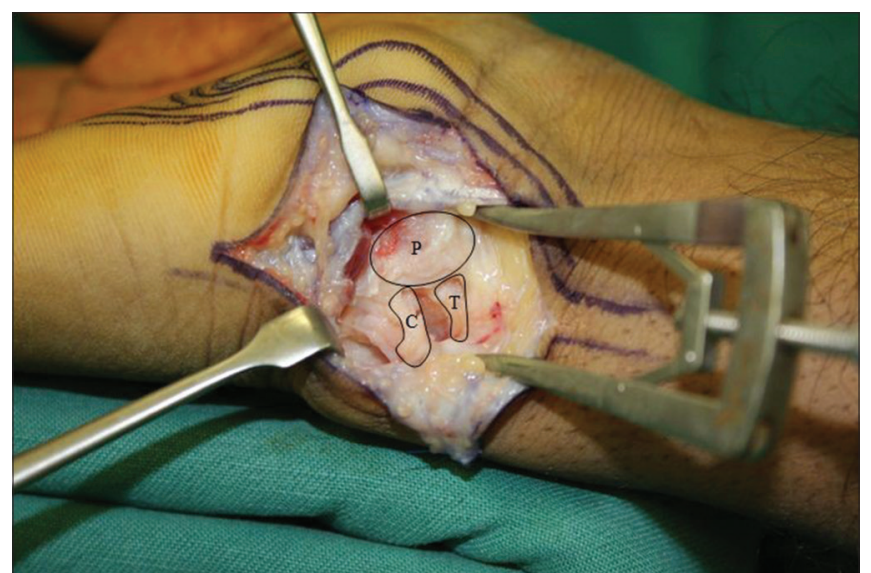

Fig. 4 Intraoperative photograph shows the wrist. C: capsule with triquetrum fragment; $\mathrm{P}$ : pisiform; T: triquetral fracture

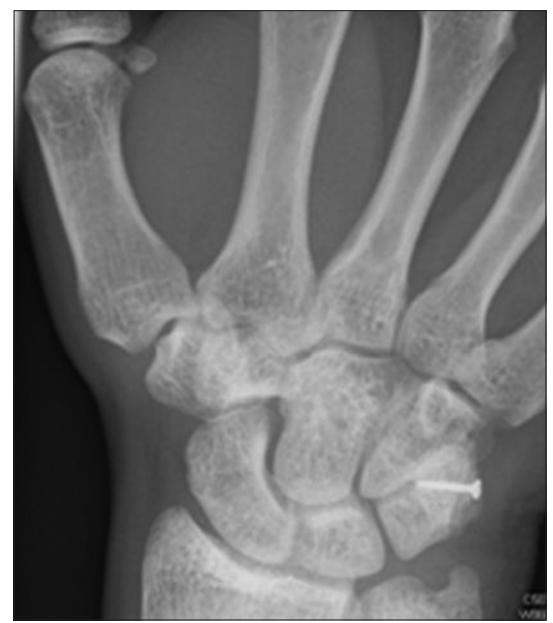

Fig. 5 Postoperative radiograph taken at the three-month follow-up shows that the fracture has healed.

Our case report presents a rare pattern of triquetral fracture with pisiform and FCU subluxation. Suzuki et al described a 21-year-old woman who fell on an outstretched hand while snowboarding and sustained a similar pattern of injury. ${ }^{(9)}$ In our case, disruption of the pisotriquetral joint was secondary to a triquetral fracture during acute wrist dorsiflexion injury. Most of the dorsoulnar joint structures were attached to the fracture fragment. 
Subluxation of the FCU is a dynamic finding that can be reproduced with the wrist in extension and ulnar deviation. With the wrist in a neutral position, there is no subluxation of the FCU. This implies the need to assess for a deforming force in cases where there is a similar fracture pattern causing subluxation. Diagnosis is primarily achieved through clinical examination using CT and MR imaging, which can confirm the configuration of the fracture injury.

Nondisplaced fractures of the triquetral body are treated via immobilisation in a short arm cast for about three to six weeks until clinical and radiographic signs of union are established. Surgery is recommended to achieve reduction and bony stability in triquetral body fractures that are displaced or have concomitant ligamentous injury. ${ }^{(10)}$ For our patient, treatment options included immobilisation after closed reduction, surgical fixation of the triquetral fracture and pisiform resection. ${ }^{(11,12)}$ It is possible to manage the fracture nonsurgically by immobilising it in slight flexion, but nonunion is a possibility, especially since the patient's presentation was delayed by two months. We opted for surgical fixation, as the fracture was displaced with intra-articular involvement and unstable with subluxation. Surgical fixation would stabilise the pisiform and FCU, with restoration of the articular surface. The patient was also significantly symptomatic, and at presentation two months post injury, the fracture had not shown signs of healing. The dorsoulnar ligaments of the pisotriquetral joint was largely intact and attached to the fracture fragment. Reduction of the fracture resulted in joint reduction.

Excision of the pisiform was not considered for our patient, because it would not have addressed the issue of the unstable FCU subluxation, as evidenced in a case reported by Suzuki et al. ${ }^{(9)}$ It is also an irreversible procedure that may weaken the supporting structures of the flexor retinaculum. In manual workers who require high grip strengths, excision of the pisiform may not be the ideal choice of treatment. A biomechanical study involving nine cadaveric wrists demonstrated that greater FCU forces (up to $29 \%$ greater than in the uninjured wrist) were required to have the same wrist motion after pisiform excision. ${ }^{(13)}$ This was because pisiform excision results in FCU tendon retraction during wrist movements, thus leading to elimination of the moment arm function, generating a significantly greater FCU force. ${ }^{(13)}$

A triquetral fracture with associated subluxation of the pisiform and FCU tendon is not commonly seen. It is crucial for the attending physician to recognise this pattern of triquetral fracture, especially if the patient presents with a snapping FCU tendon. Open reduction and internal fixation typically results in a good outcome.

\section{REFERENCES}

1. Höcker K, Menschik A. Chip fractures of the triquetrum. Mechanism, classification and results. J Hand Surg Br 1994; 19:584-8.

2. De Beer JD, Hudson DA. Fractures of the triquetrum. J Hand Surg Br 1987; 12:52-3.

3. Garcia-Elias M. Dorsal fractures of the triquetrum-avulsion or compression fractures? J Hand Surg Am 1987; 12:266-8.

4. Kubiak R, Slongo $\mathrm{T}$, Tschäppeler $\mathrm{H}$. Isolated dislocation of the pisiform: an unusual injury during a cartwheel maneuver. J Trauma 2001; 51:788-9.

5. Moojen TM, Snel JG, Ritt MJ, et al. Pisiform kinematics in vivo. J Hand Surg Am 2001; 26:901-7.

6. Oh SK, Seong PC, Ho YW. Acute Isolated Pisiform Dislocation - A Case Report. J Korean Orthop Assoc 2007; 42:688-91.

7. Immermann EW. Dislocation of the pisiform. J Bone Joint Surg Am 1948; 30A:489-22

8. Ishizuki M, Nakagawa T, Itoh S, Furuya K. Positional dislocation of the pisiform. J Hand Surg Am 1991; 16:533-5.

9. Suzuki T, Nakatsuchi Y, Tateiwa Y, Tsukada A, Yotsumoto N. Osteochondral fracture of the triquetrum: a case report. J Hand Surg Am 2002; 27:98-100.

10. Green DP, O'Brien ET. Open reduction of carpal dislocations: indications and operative techniques. J Hand Surg Am 1978; 3:250-65.

11. McCarron RF, Coleman W. Dislocation of the pisiform treated by primary resection. A case report. Clin Orthop Relat Res 1989; (241):231-3.

12. Schädel-Höpfner $M$, Böhringer $G$, Junge A. Dislocation of the pisiform bone after severe crush injury to the hand. Scand J Plast Reconstr Surg Hand Surg 2003; 37:252-5.

13. O'Keefe KD, Werner FW, Boyette $M$, et al. Effect of pisiform excision or pisotriquetral arthrodesis as a treatment for pisotriquetral arthritis: a biomechanical study. J Hand Surg Am 2013; 38:1913-8. 